gradual decline in glomerular filtration rate (Figure). These renal changes result in a reduced ability of the elderly patient to excrete administered drugs and also to excrete normally metabolized waste body products. For example, creatinine, a normal metabolite of muscle creatinine has a reduced excretion rate in the elderly patient. However, despite a decline in glomerular filtration rate, healthy elderly patients have the same circulating level of serum creatinine as younger patients because they have less skeletal muscle and, therefore, less muscle metabolism and creatinine production. If serum creatinine leveis are elevated in an elderly patient, this implies a severe decrement in renal function, greater than the expected agerelated decline.

Most administered anaesthetic agents are highly lipid soluble. Lipid soluble drugs filtered by the glomeruli are readily reabsorbed by renal tubules and therefore are not excreted. The liver converts lipid soluble drugs by glucuronidation or other metabolic processes into water soluble metabolites which, after filtration by the glomeruli, are reabsorbed less readily by the renal tubules and excreted. As a result of the aging process, the ability to both metabolize and excrete anaesthetic drugs is impaired. For example, the elimination half life $\left(t_{1} \beta\right)$ of fentanyl is 265 minutes in young patients compared to 945 minutes in elderly patients. The reduced clearance of drugs in the elderly patient also affects muscle relaxants, which suggests muscle relaxants should be administered at less frequent intervals than in younger patients.

\section{Anaesthetic implications}

The effect of anaesthetic drugs in elderly patients is usually exaggerated due to a reduced volume of distribution and reduced protein binding. The reduced CNS function in elderly patients may also contribute to their greater sensitivity to intravenous and inhalational anaesthetic agents. As a result, the anaesthetic must, in general, reduce the dose of administered intravenous agents in the elderly patient. Since accumulation of fat-soluble drugs is enhanced due to a higher percentage of body fat, lipid soluble drugs may be expected to have a longer duration of action in these patients. Because the basal metabolic rate and hepatic and renal functions are reduced, metabolism and elimination of anaesthetic drugs will be impaired and again a longer duration of action may be expected.

Cardiac output is reduced. We should therefore expect a delayed onset of action following administration of intravenous agents and a more rapid onset of action with inhalational anaesthetics. Intravenous drugs must be administered not only in reduced dosages but, because of reduced clearance should also be reinjected less frequently. Finally, because of lower resting arterial $\mathrm{PO}_{2}$ values and higher closing volumes, attention to pulmonary toilet and ventilatory assistance postoperatively is crucial in geriatric patients. Similarly, they may require a higher $\mathrm{FIO}_{2}$ when undergoing operative procedures than do younger patients undergoing similar procedures.

\section{Bibliography}

Bentley $J B$, Borel JD, Nenad RE. Influence of age on the pharmacokinetics of fentanyl. Anesth Analg 1982; 61 : 171-2.

Evars $T$. The physiological basis of geriatric anaesthesia. Anaesth Intensive Care 1973; 1: 3]9-22.

Greenblatt $D J$. Reduced serum albumin concentration in the elderly: a report from the Boston collaborative drug survcillance program. J Am Ger Soc 1979; 27: 20-2.

McLeod K. Hull CJ, Watson MJ. Effects of aging on the pharmacokinetics of pancuronium. Br J Anaesth 1979; 51: 435-8.

McLeskey CH. Anesthesia for the Geriatric Patient. In: Advances in Anesthesia. Volume 2, eds. Stoelting RK, Barash PG, Gallagher TJ. Year Book Medical Publishers, Chicago, 1985; pp 31-68.

Pontoppidan H. Beecher HK. Progressive loss of protective reflexes in the airway with the advance of age. JAMA 1960; 174: $2209-13$

Pontoppidan $H, G e f f i n ~ B$, Lowenstein $E$. Acute respiratory failure in the adult. N Engl J Med 1972; 287: 690-8

Sievens WC, Dolan WM, Gibbons RT et al. Minimum alveolar concentrations (MAC) of isoflurane with and without nitrous oxide in paticnts of various ages. Ancsthesiology 1975; 42: $197-200$.

\section{Changes in drug disposition}

It is of interest that the chapter on alterations in drug disposition in a recently published textbook on geriatric anaesthesia deals almost exclusively with systemically administered drugs, and not much with inhalational agents. ${ }^{\prime}$ The whole problem of changes in disposition of inhalational agents is an intriguing one, and the demonstration that minimum alveolar concentrations (MAC) of isoflurane and halothane decrease with age highlights the difficulty of differentiating pharmacokinetic differences from pharmacodynamic differences. ${ }^{2,3}$ If an age-related difference in drug response is shown to exist, it must be demonstrated that changes in drug disposition are not important before changes in drug effect can be inferred. 


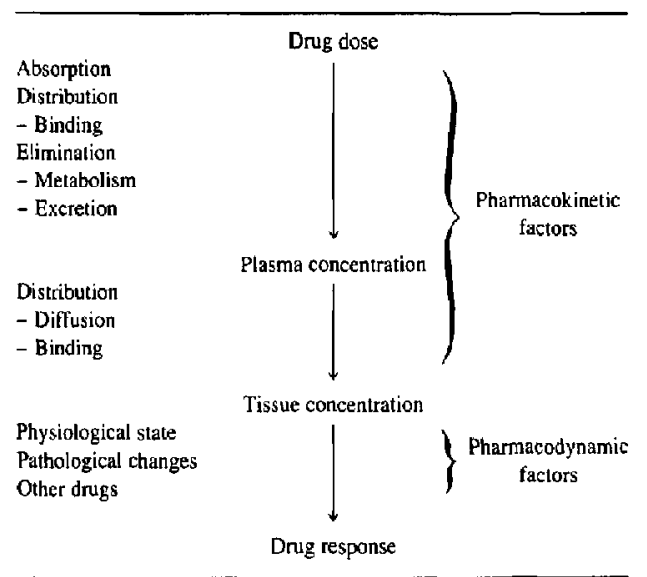

FGURE Elements linking drug dose to drug response

It appears to have been assumed that there are no real differences in the disposition of gaseous anaesthetics between young and old patients, but the recent finding by our unit that the blood:breath coefficient for alcohol is substantially higher for normal older individuals suggests that pharmacokinetic changes cannot be ignored for inhalational agents. ${ }^{4}$

This review will follow the standard pharmacokinetic format outlined in the Figure. It should be noted that although changes in drug absorption, distribution and elimination may be used to predict changes in plasma concentration reasonably well, unless alterations in disposition in the relevant tissues are known, changes in receptor concentration may not be appreciated. At present, very little definitive information on age-related differences in distribution within relevant organ systems is avaiiable.

Absorption of drugs from the gastrointestinal tract is usually not affected by old age, which is understandable when it is recalled that most drugs are absorbed by passive diffusion. Profound changes in gut physiology occur with age but these will only modify the transport of those agents requiring active Iransport mechanisms for absorption,

Drug distribution, in contrast, may be quite different in older patients. The practical message to be stressed is that the elderly are generally smaller than their younger counterparts, and require dosage adjustments on that basis alone. But substantial differences in distribution can be shown for specific drugs, with lipid solubility a critical determinant. The alterations in body structure and function that accompany normal aging result in a decrease in lean tissue and body water and an increase in adipose tissue in relation to total body weight. ${ }^{5}$ Measurements of volumes of distribution would be expected to increase with age for more lipophilic drugs and to decrease for less lipaphilic drugs.

An age-related increase in the $\mathrm{Vd}$ of diazepam was found by Klotz et al., ${ }^{6}$ in conjunction with an increase in plasma half-life, $t_{1}$. But clearance was not much different in older subjects, since these changes were roughly proportional (since clearance $=\mathrm{Ke} \times \mathrm{Vd}=0.693 \mathrm{Vd} / \mathrm{t}_{1}$ ). This helps explain our results for morphine, a less lipophilic drug, for which a decrease in Vd was coupled with a decrease in plasma $t_{1}$ while the clearance changed very little. ${ }^{7}$

Binding of drugs to albumin and other plasma proteins can be altered in older patients. Many drugs may be less bound because albumin concentrations tend to decrease with age, but free drug concentrations may not be much different. ${ }^{8}$ of more concern is the recent study by Paxton and Briant indicating that the concentrations of $\alpha_{1}$-acidglycoprotein increase with aging, and that the free concentrations of basic drugs such as lidocaine or propranolol may be markedly reduced in both sick and healthy elderly patients. ${ }^{9}$

Drug metabolism may be altered by age for some agents, but overall, environmental, nutritional and genetic influences are more important than age in modifying hepatic biotransformation. Conjugative (phase II) reactions are generally preserved in older patients, but some oxidative (phase I) reactions are impaired, although not in a predictable fashion. The study by Vestal et al. is interesting because they found minimal age-related change in the intrinsic hepatic clearance of propranolol, although cigarette smoking was associated with a decrease in oral propranolol clearance with age. ${ }^{10}$ Of significance is the further work by this group concerning the effects of propranolol, so that by demonstrating an age-related decrease in sensitivity of $\beta$-adrenoreceptors to both isoproterenol and propranolol, they were able to show a phamacody. namic difference in the absence of a pharmacokinetic change. ${ }^{11}$

In this context the interference by cimetidine in the metabolism of many drugs becomes important, not because it occurs only in older patients, but because the phenomenon of polypharmacy makes this kind of interaction much more likely with old age. The concomitant use of cimetidine and drugs active on the central nervous system would be expected to be more dangerous in the elderly. ${ }^{12}$

Drug excretion declines with tubular and glomerular function as the years progress. ${ }^{13}$ The mean loss in glomerular filtration rate by age 65 is 35 per cent, and this kind of decrease applies not only to those drugs eliminated unchanged in the urine, such as digoxin or the aminogly- 
cosides, but as well to some drugs whose active metabolites are excreted by the kidney, such as acctylprocainamide $^{14}$ and normepcridine. ${ }^{15}$

Review of the data on age-related changes in renal function leads to a final message conceming older patients who come under our care. There are substantial differences in renal function between older patients (and by implication, with regard to a variety of other functions). Chronologic age is a notoriously poor indicator of biologic age, and each patient must be regarded as an individual system when age-related changes in drug disposition are considered. No formula solutions can be considered applicable to all elderly, because the problems of each person are unique.

\section{References}

1 Schmucker $D L$. Alterations in dng disposition. $I n$ : Geriatric anesthesia: principles and practice. Stephen $C R$, Assaf $A E$. eds. Stoneham, Butterworths, 1986; 155-88.

2 Stevens WC, Dolan WM, Gibbens RT et al. Minimum alveolar concentrations (MAC) of isoflurane with and without nitrous oxide in patients of varying ages. Anesthesiology 1975; 42: 197-200.

3 Quasha AL. Eger EJ. Tinker JM. Determinations and applications of MAC. Anesthesiology 1983; 315-34.

4 Sitar DS. Wilson A, Mailoy WD, McCarthy D. Aging, pulmonary disease and the estimation of blood alcohol concentration by analyses of breath samples. Alcoholism: Clin Exper Res. In Press. 1987

5 Bruce A, Andersson M, Arvidsson B, Isaksson B. Body composition: prediction of nomal body potassium, body water and body fat in adults on the basis of body height, body weight and age. Scand J Clin Lab lnvest 1980; 40: $461-73$.

6 Klotz U, Avant GR, Hoyunna A, Schenker S, Wilkinson $G R$. The effects of age and liver disease on the disposition and elimination of diazepam in adult man. J Clin Invest 1975; 55: 347-59.

7 Owen J, Sitar D, Berger L, Brownell L, Duke P, Mitenko $P$. Age-related morphine kinetics. Clin Pharmacol Ther 1983; 34: 364-8.

8 Greenblatt DJ, Sellers EM, Shader RI. Drug disposition in old age. N Engl J Med 1981; 306: $1081-8$.

9 Paxton $J W$, Briamt $R H$. $\alpha-1$ acid glycoprotein concentrations and propanolol binding in elderly patients with acute illness. Br J Clin Pharmacol 1984; 18: 806-10.

10 Vestal RE, Wood AJJ, Branch RA, Shand DG. Wilkinson $G R$. Effects of age and cigarette smoking on propanolol disposition. Clin Pharmacol Ther 1979; 26: 8-15.

11 Vestal RE, Wood A.IJ, Shand DG. Reduced $\beta$-idreno receptor sensitivity in the elderly. Clin Pharmacol Ther 1979; 26: $181-6$.
12 Greenblatt DJ, Abernathy DR, Morse BA, Harmatz BA, Shader RI. Clinical importance of the interaction of diazepam and cimetidine. N Engl J Med 1984; 310: 1639-43.

13 Rowe JW, Andres $R$, Tobin JD. Norris AH, Shock NW. The effects of age on creatinine clearance in man: a crosssectional and longitudinal study. J Gerontol 1976; 31: 155-63.

14 Reidenberg MM, Camacho $M$, Kiuger I, Drayer DE. Aging and renal clearance of procainamide and acetylprocainamide. Clin Pharmacol Ther 1980; 28: 732-5.

15 Szeto HH, Inturrigi CE, Houde R, Saal S, Chergh J, Reidenberg $M M$. Accumulation of normeperidine, an active metabolite of meperidine, in patients with renal failure or cancer. Ann Int Med 1977; 86: 738-41.

\section{Cardiac risk assessment and reduction in the elderly}

Cardiovascular complications are a significant and potentially ayoidable source of perioperative morbidity and mortality in the elderly. ${ }^{1}$ Ideally, preoperative risk assessment will identify a high-risk subgroup of patients in which a variety of strategies aimed at risk reduction can be employed. Table I lists severa] indices of the risk of major perioperative cardiovascular complications. Some indices are obtainable from a history, physical examination, and routine laboratory investigations, whereas others are relatively expensive or invasive. Table II reports findings regarding the sensitivity, specificity, and overall predictive value of the indices listed in Table $I$, as well as details of experimental design and statistical analysis. In general, conclusions from large prospective studies employing a multivariate statistical approach should be given most credence. Most readily available indices lack either the sensitivity or specificity necessary to precisely identify a subgroup of patients at high risk. Other more powerful indices are too expensive for widespread application as screening tools. Available data do not allow description of a cost-effective technique by which currently available indices of risk can be sequentially applied to comprehensively screen a large geriatric surgical population. Furthermore, none of the available strategies for risk reduction have been prospectively demonstrated to alter perioperative cardiac morbidity or mortality in patients at risk. Much further research will be necessary before 